\title{
BMJ Open Risk factors of direct heat-related hospital admissions during the 2009 heatwave in Adelaide, Australia: a matched case-control study
}

\author{
Ying Zhang, ${ }^{1}$ Monika Nitschke, ${ }^{2}$ Antoinette Krackowizer, ${ }^{3}$ Keith Dear, ${ }^{4}$ \\ Dino Pisaniello, ${ }^{3}$ Philip Weinstein, ${ }^{5}$ Graeme Tucker, ${ }^{2}$ Sepehr Shakib, ${ }^{6}$ Peng $\mathrm{Bi}^{3}$
}

To cite: Zhang Y, Nitschke M, Krackowizer A, et al. Risk factors of direct heat-related hospital admissions during the 2009 heatwave in

Adelaide, Australia: a matched case-control study. BMJ Open 2016;6: e010666. doi:10.1136/ bmjopen-2015-010666

- Prepublication history for this paper is available online. To view these files please visit the journal online (http://dx.doi.org/10.1136/ bmjopen-2015-010666)

Received 27 November 2015 Revised 5 May 2016 Accepted 6 May 2016

CrossMark

For numbered affiliations see end of article.

\section{Correspondence to}

Dr Ying Zhang;

ying.zhang@sydney.edu.au

Dr Peng Bi;

peng.bi@adelaide.edu.au

\section{ABSTRACT}

Objective: The extreme heatwave of 2009 in South Australia dramatically increased morbidity, with a 14-fold increase in direct heat-related hospitalisation in metropolitan Adelaide. Our study aimed to identify risk factors for the excess morbidity.

Design: A matched case-control study of risk factors was conducted.

Setting: Patients and matched community controls were interviewed to gather data on demographics, living environment, social support, health status and behaviour changes during the heatwave.

Participants: Cases were all hospital admissions with heat-related diagnoses during the 5-day heatwave in 2009. Controls were randomly selected from communities.

Outcome measures: Descriptive analyses, simple and multiple conditional logistic regressions were performed. Adjusted ORs (AORs) were estimated.

Results: In total, 143 hospital patients and 143 matched community controls were interviewed, with a mean age of 73 years (SD 21), 96\% European ethnicity, $63 \%$ retired, $36 \%$ with high school or higher education, and $8 \%$ institutional living. The regression model indicated that compared with the controls, cases were more likely to have heart disease $(\mathrm{AOR}=13.56,95 \% \mathrm{Cl}$ 1.27 to 144.86) and dementia (AOR=26.43, 95\% Cl 1.99 to 350.73$)$. The protective factors included higher education level ( $A O R=0.48,95 \% \mathrm{Cl} 0.23$ to 0.99 ), having air-conditioner in the bedroom (AOR=0.12, 95\% Cl 0.02 to 0.74 ), having an emergency button (AOR=0.09, $95 \%$ $\mathrm{Cl} 0.01$ to 0.96$)$, using refreshment $(\mathrm{AOR}=0.10,95 \% \mathrm{Cl}$ 0.01 to 0.84 ), and having more social activities (AOR=0.11, 95\% Cl 0.02 to 0.57).

Conclusions: Pre-existing heart disease and dementia significantly increase the risk of direct heat-related hospitalisations during heatwaves. The presence of an air-conditioner in the bedroom, more social activities, a higher education level, use of emergency buttons and refreshments reduce the risk during heatwaves.

\section{INTRODUCTION}

The newly released Intergovernmental Panel on Climate Change (IPCG) 5th Assessment

\section{Strengths and limitations of this study}

- This is the first matched case-control study using collected individual data to explore risk factors of heat-related hospitalisations in Australia.

- A broad range of potential risk factors were investigated.

- The exposure of heatwave in Adelaide was very unique with dramatically increased morbidity.

- Data quality was ensured by using patients' medical record data collected from hospitals as well as questionnaires.

- The major limitation might be from only recruiting $60 \%$ of eligible patients.

Report has once again confirmed the unequivocal global warming trend over the past century. In particular, it confirms that extreme heat events are very likely to happen with a higher frequency and longer duration. ${ }^{1}$ Australia's climate is already changing, with significant increases in heat extremes observed since the 1960s. Australian summers in recent years have been extremely hot, including the record-breaking Australian summer of 2009, 2013 and $2014,{ }^{2}$ with potentially significant public health implications.

Evidence on the adverse impacts of heatwaves on human health has been gathered including increased morbidity in many regions. ${ }^{3}$ For example, extreme heat has been reported to be associated with a $3 \%$ increase in all-cause hospitalisation in older people in the USA. ${ }^{4}$ Our previous studies have reported that heatwaves in Adelaide are associated with increased morbidity for renal ischaemic, mental health and direct heat-related illnesses, ${ }^{5-7}$ affecting various vulnerable populations including the elderly, culturally and linguistically diverse (CALD) communities, remote communities and outdoor workers. ${ }^{8-11}$

There is a need to develop appropriate public health interventions to reduce the 
negative health impacts from heatwaves. ${ }^{12}$ However, the risk factors for vulnerability to heatwaves at both individual (eg, health status and living conditions) and community (eg, social support and health services) levels have not yet been investigated in depth. ${ }^{13}$ The majority of previous studies on heat and health have focused on identifying and quantifying the relevant health outcomes and their associations with heatwaves at a population level, with only a few investigating associated risk factors using individual data. ${ }^{4}{ }^{13-15}$ A negative relationship between social capital, health and adaptation to climate change has been recently identified among older people in the UK, suggesting a complex relationship between potential risk factors and vulnerability. ${ }^{16}$ However, inconsistent findings have been reported from previous studies. For instance, women are at a higher risk in most of the European countries, but men are more vulnerable in heatwaves in the USA. ${ }^{17}$ Moreover, most of the previous studies have focused on mortality only and had a relatively narrow focus on some of the potential risk factors, for example, health status, behaviours, socioeconomic status or living conditions. ${ }^{17-20}$

In Australia, a very limited number of studies have assessed risk factors for heatwave-related mortality and morbidity, with no previous case-control studies being conducted. ${ }^{21}$ The 2009 heatwave in Southern Australia provided an excellent opportunity to implement a study focusing on morbidity during an extreme heatwave using such a study design, and thereby improving the quality of epidemiological data available on risk factors. Our study, drawing on individual data, aimed to provide robust evidence on risk factors for heat-related conditions in the Australian context to support relevant policy and intervention programmes, including refining current heat and health early warning systems, and developing better individual health services that could reduce morbidity during heatwaves.

\section{METHODS}

\section{Study design and population}

A case-control study was conducted in Adelaide in 2012-2013, targeting vulnerable groups from the 2009 heatwave. Adelaide, the capital city of South Australia, is located coastally in the middle south of Australia with a total population of 1.2 million in 2012. It has a semi-arid climate with hot daytime temperatures in summer and cooler nights. In 2009, Adelaide experienced a severe heatwave from 26 January to 7 February, a 13-day episode, with six consecutive days having maximum temperatures over $40^{\circ} \mathrm{C}$. The maximum temperature soared to $45.7^{\circ} \mathrm{C}$ on 28 January and was followed by an unusually high minimum temperature of $34^{\circ} \mathrm{C}$ the following night. The health impacts of the 2009 Adelaide heatwave included a $9.5 \%$ increase in mortality, an almost 14-fold increase in direct heat-related hospital admissions, and a 2.4\% increase in total emergency department visits. ${ }^{5}$ Furthermore, our previous report found that mortality and morbidity peaked during the five hottest days, 28 January to 1 February. ${ }^{22}$ The maximum temperatures of these days were $45.7^{\circ} \mathrm{C}, 43.4^{\circ}$ $\mathrm{C}, 43.1^{\circ} \mathrm{C}, 41.1^{\circ} \mathrm{C}$ and $40.6^{\circ} \mathrm{C}$, respectively. The 5-day period was chosen as the heatwave exposure period of the present study.

Direct heat-related hospitalisation based on International Classification of Diseases, 10th Revision (ICD-10) codes, X30 Exposure to excessive natural heat, T67 Effects of heat and light, and E86 Volume depletion during the heatwave exposure period were treated as the adverse health outcomes in this study. ${ }^{21}$ Given the potential for underdiagnosis, the ICD-10 codes for direct heat-related hospitalisations were selected from the primary (principal) diagnosis, as well as from any of the additional diagnoses in the medical record.

\section{Participants (cases and controls)}

Cases included those who were hospitalised for direct heat-related conditions from all public hospitals in Adelaide over the 5-day exposure period. Controls were those who were exposed to the heatwave period without any heat-related hospitalisations. Controls were randomly selected from the South Australian State Electoral Roll (SASER) and were 1:1 matched to cases by age ( \pm 2 years) and gender. The SASER provides an electronic source of people who resided in metropolitan Adelaide (postcodes 5000-5199). Persons who were not living in the Adelaide metropolitan area during the 2009 heatwave exposure period were excluded.

The process of recruitment is stated as follows. Eligible cases were first contacted by an initial information letter and then a follow-up letter. A phone call would follow from the South Australian Department for Health and Ageing (DHA) if there was no response. Matched controls who were randomly selected from the SASER were first contacted by an invitation letter with project information. A follow-up letter was sent if no response was received within 2 weeks. If no response was received after the follow-up letter in one week, then a telephone call was made to contact the potential participants. The random selection was repeated if no response was reached after this initial procedure.

\section{Ethical approvals and recruitment}

In order to collect patients' data from the hospitals' medical records, ethics approvals were also obtained from relevant hospital ethics committees. All personal information was removed before the data were passed on for analysis.

\section{Data collection}

Questionnaire: Risk factors were investigated using a specifically designed questionnaire, which was developed on the basis of a literature review and informed by other studies undertaken by our team. ${ }^{5-11}$ The questions were related to factors including environmental conditions, health status, individual behaviours and demographic 
information. Physical impairment was measured using the Activities of Daily Living (ADL) scale. Participants were asked to answer the frequency of having social activities (eg, going to church or community-organised events) by selecting from the following three options '(1) never (2) less than once a week and (3) more than once a week'. Consistent face-to-face or telephone interviews of controls and cases were conducted by an experienced interviewer who had been trained for this specific project.

Environmental data: Daily maximum temperature and relative humidity between January and February 2009 were obtained from the Australian Bureau of Meteorology Kent Town weather station (ID: 023090). It is located about $2 \mathrm{~km}$ east of central Adelaide city. It is the only weather observation station that has meteorological data representative of conditions in the Adelaide metropolitan area. During the 2009 heatwave, relative humidity stayed below $40 \%$ and no rainfall was recorded.

Hospital records: Morbidity data were obtained from the South Australian DHA, including the Integrated South Australian Activity Collection (ISAAC) and the Emergency Department Data Collection (EDDC). Patients' case-note data for admissions in 2009 and earlier were retrieved from relevant hospitals.

\section{Statistical power}

The power calculation was based on McNemar's test, the method of Dupont for matched case-control studies. ${ }^{23}$ We assumed that $30 \%$ of the control subjects were exposed to any risk factor and defined the significance level at 0.05 . Our sample could provide a statistical power of $80 \%$ to detect a minimum OR of 2.0 .

\section{Statistical analyses}

First, a descriptive analysis was performed. Characteristics of the cases and controls and the distribution of potential risk factors were described. A Student's t-test or $\chi^{2}$ test was applied to examine the association of any potential risk factor and morbidity. The variables significantly associated with the outcomes $(p<0.05)$ were entered into the multivariate regression model. Conditional logistic regression models were applied to examine any interaction between potential risk factors and morbidity. ORs and adjusted ORs (AORs) and their 95\% CIs were calculated.

\section{RESULTS}

\section{Characteristics of participants}

In total, there were 298 patients admitted to the public hospitals over the study exposure period. Among these patients, 31 deceased, 25 under 18 years and 3 aboriginal patients were excluded, leading to 239 eligible cases to recruit. We interviewed 143 cases $(60 \%$ of the total eligible patients) and 143 matched community controls (table 1). For the total sample of cases and controls, the mean age was 73 years (SD 21), 96\% of European ethnicity; $63 \%$ were retired, $36 \%$ completed high school or higher education, $8 \%$ lived in institutions, and $30 \%$ were still in the workforce. For those who did not participate, $39 \%$ were female with a mean age of 69 years (SD $25)$. The main reason for not being able to participate (90\% of the non-participants) was due to their incomplete hospital records or unknown address so that we could not reach them. The reason for cases refusing to participate included three patients with dementia, six who died close to the interview dates (not recorded in the collected hospital record data) and one non-English speaking case.

Cases and controls were significantly different in some aspects of the characteristics, especially with respect to their living conditions, their support from family and community and health status. There were no obvious differences in terms of behaviour changes during heatwaves except for the use of air-conditioner $(\mathrm{p}<0.0001)$.

\section{Single-variable analysis}

The results of the single-variable analysis with a $\mathrm{p}$ value $<0.05$ are shown in table 2 , as used in the subsequent multiple regression analysis. It shows that many factors might contribute to the direct heat-related morbidity, with kidney disease $(\mathrm{OR}=8.9)$, dementia $(\mathrm{OR}=8.3)$ and heart disease $(\mathrm{OR}=6.3)$ being the most significant risk factors, without controlling for any other factors.

\section{Multiple regression and AORs}

AORs of those variables entered into multiple regressions are shown in table 2. The variables significantly included in the final conditional logistic regression model and the AOR are shown in table 3 with an $\mathrm{R}^{2}=0.85$. The protective factors included having a higher education level (AOR $=0.48,95 \%$ CI 0.23 to 0.99 ), having air-conditioning in the bedroom $(\mathrm{AOR}=0.12,95 \%$ CI 0.02 to 0.74 ), having an emergency button (AOR $=0.09$, $95 \%$ CI 0.01 to 0.96 ), using refreshment (AOR $=0.10$, 95\% CI 0.01 to 0.84 ), and having more social activities (AOR $=0.11,95 \%$ CI 0.02 to 0.57 ). Factors that were likely to increase the risk included pre-existing heart disease (AOR=13.56, 95\% CI 1.27 to 144.86) and dementia (AOR=26.43, 95\% CI 1.99 to 350.73 ).

\section{DISCUSSION}

This is the first case-control study comprehensively investigating a wide range of potential factors contributing to heat-related morbidity in Australia. We have identified factors that could significantly increase or reduce the risk of direct heat-related hospitalisations during heatwaves. These findings will contribute further to existing relevant policies and interventions that have been implemented since the 2009 heat event, so as to prevent and reduce heat-related morbidity during extreme heat events. 
Table 1 Characteristics of the participants and simple comparison between cases and controls

\begin{tabular}{|c|c|c|c|}
\hline Total $\mathbf{N}=\mathbf{2 8 6}$ & $\begin{array}{l}\text { Cases } \\
143\end{array}$ & $\begin{array}{l}\text { Controls } \\
143\end{array}$ & p Value* \\
\hline \multicolumn{4}{|l|}{ Demographic } \\
\hline Female & $50 \%$ & $50 \%$ & \\
\hline Mean age & 73 (SD 21) & $73(\mathrm{SD} 21)$ & \\
\hline European ethnic & $94 \%$ & $97 \%$ & 0.06 \\
\hline Married/de facto & $43 \%$ & $60 \%$ & $<0.01$ \\
\hline Highest education level more than year 12 & $8 \%$ & $41 \%$ & $<0.01$ \\
\hline Annual personal income $>\$ 50000$ & $4 \%$ & $27 \%$ & $<0.01$ \\
\hline Working & $27 \%$ & $30 \%$ & 0.10 \\
\hline \multicolumn{4}{|l|}{ Living conditions } \\
\hline Institutional living & $6 \%$ & $10 \%$ & 0.18 \\
\hline Single-level independent house living & $69 \%$ & $68 \%$ & 0.10 \\
\hline North-facing building & $78 \%$ & $56 \%$ & $<0.01$ \\
\hline Living alone & $43 \%$ & $33 \%$ & 0.08 \\
\hline Having a pet & $27 \%$ & $44 \%$ & $<0.01$ \\
\hline Air-conditioner in the house & $51 \%$ & $87 \%$ & $<0.01$ \\
\hline Air-conditioner in the bedroom & $17 \%$ & $52 \%$ & $<0.01$ \\
\hline Easy ventilation & $43 \%$ & $82 \%$ & $<0.01$ \\
\hline \multicolumn{4}{|l|}{ Support } \\
\hline Having private health insurance & $17 \%$ & $64 \%$ & $<0.01$ \\
\hline Having an emergency button & $11 \%$ & $31 \%$ & $<0.01$ \\
\hline Having social activities more than once a week & $30 \%$ & $89 \%$ & $<0.01$ \\
\hline No daily access to media & $13 \%$ & $2 \%$ & $<0.01$ \\
\hline \multicolumn{4}{|l|}{ Health status } \\
\hline Heart disease/symptoms & $49 \%$ & $15 \%$ & $<0.01$ \\
\hline Asthma & $12 \%$ & $12 \%$ & 0.10 \\
\hline Respiratory disease/symptoms & $30 \%$ & $18 \%$ & 0.01 \\
\hline High blood pressure & $47 \%$ & $37 \%$ & 0.093 \\
\hline Kidney disease/symptoms & $33 \%$ & $5 \%$ & $<0.01$ \\
\hline Diabetes & $30 \%$ & $11 \%$ & $<0.01$ \\
\hline Dementia & $19 \%$ & $4 \%$ & $<0.01$ \\
\hline Depression/symptoms & $42 \%$ & $13 \%$ & $<0.01$ \\
\hline Liver disease & $15 \%$ & $3 \%$ & $<0.01$ \\
\hline Neurological disease & $16 \%$ & $6 \%$ & $<0.01$ \\
\hline Cancer & $19 \%$ & $4 \%$ & $<0.01$ \\
\hline Taking 5 or more medicines & $56 \%$ & $39 \%$ & 0.013 \\
\hline Confine to bed & $41 \%$ & $0.7 \%$ & $<0.01$ \\
\hline Need assistance for daily activities $A D L=1$ & $81 \%$ & $1.4 \%$ & $<0.01$ \\
\hline \multicolumn{4}{|l|}{ Behaviour changes during a heatwave } \\
\hline Stop activities during the day & $46 \%$ & $54 \%$ & 0.23 \\
\hline Take extra shower or bath & $14 \%$ & $19 \%$ & 0.26 \\
\hline Use an air-conditioner & $45 \%$ & $85 \%$ & $<0.01$ \\
\hline Go out during a heatwave & $42 \%$ & $40 \%$ & 0.68 \\
\hline Open windows & $20 \%$ & $17 \%$ & 0.58 \\
\hline
\end{tabular}

${ }^{*} p$ Value: comparing between cases and controls.

ADL, Activities of Daily Living.

One striking finding from our study is the beneficial factor of having more social support in reducing the morbidity during heatwaves. Social isolation was also identified as a key risk factor of heat-related deaths during heatwaves in earlier studies. ${ }^{15}$ Usage of emergency buttons (which is usually linked with community health professionals) means vulnerable people could receive timely health support when they are in need. This may contribute significantly to the reduction of morbidity during heatwaves, and should be recommended to high-risk groups if conditions permit. Our results suggest that by regularly checking high-risk groups during heat, or by providing heat-health related information to the community and offering social support and timely interventions during extreme heat, vulnerable groups are more likely to achieve better health outcomes during heatwaves.

We could not find many risk differences between cases and controls when considering individual behavioural changes during the 2009 heatwave, except for the use of refreshment during heat. In addition to vulnerability, there may be a range of factors affecting how people 
Table 2 ORs of the risk factors by simple logistic regression and AORs by multiple regressions ${ }^{*}$

\begin{tabular}{|c|c|c|c|c|c|c|}
\hline Factors & ORs & SE & p Value & AORs & SE & p Value \\
\hline Married/de facto & 0.46 & 0.12 & $<0.01$ & 0.97 & 0.18 & 0.89 \\
\hline Institutional living & 0.54 & 0.25 & 0.19 & 0.17 & 0.20 & 0.14 \\
\hline Higher income level & 0.34 & 0.08 & $<0.01$ & 0.87 & 0.35 & 0.73 \\
\hline North-facing building & 2.78 & 0.76 & $<0.01$ & 1.01 & 0.59 & 0.99 \\
\hline Living alone & 1.80 & 0.50 & 0.04 & 1.98 & 1.25 & 0.28 \\
\hline Having a pet & 0.44 & 0.12 & $<0.01$ & 0.56 & 0.32 & 0.31 \\
\hline Higher education level & 0.27 & 0.06 & $<0.01$ & 0.48 & 0.18 & 0.05 \\
\hline Air-conditioner in house & 0.15 & 0.05 & $<0.01$ & 0.46 & 0.28 & 0.31 \\
\hline Air-conditioner in bedroom & 0.18 & 0.06 & $<0.01$ & 0.12 & 0.11 & 0.02 \\
\hline Easy ventilation & 0.18 & 0.06 & $<0.01$ & 0.41 & 0.26 & 0.16 \\
\hline Having private health insurance & 0.07 & 0.03 & $<0.01$ & 0.30 & 0.20 & 0.07 \\
\hline Having an emergency button & 0.22 & 0.08 & $<0.01$ & 0.09 & 0.11 & 0.05 \\
\hline Having daily media access & 0.16 & 0.10 & $<0.01$ & 3.06 & 3.51 & 0.33 \\
\hline Heart disease & 6.33 & 2.27 & $<0.01$ & 13.56 & 16.39 & 0.03 \\
\hline Asthma & 1.77 & 0.61 & 0.10 & 1.26 & 1.00 & 0.77 \\
\hline Respiratory disease & 1.90 & 0.52 & 0.02 & 2.36 & 1.97 & 0.30 \\
\hline High blood pressure & 1.56 & 0.40 & 0.08 & 0.88 & 0.52 & 0.83 \\
\hline Kidney diseases & 8.90 & 4.24 & $<0.01$ & 2.85 & 2.64 & 0.86 \\
\hline Diabetes & 5.50 & 2.44 & $<0.01$ & 1.36 & 1.46 & 0.77 \\
\hline Dementia & 8.33 & 5.09 & $<0.01$ & 26.43 & 34.86 & 0.01 \\
\hline Depression & 5.10 & 1.76 & $<0.01$ & 3.38 & 3.05 & 0.18 \\
\hline Neurological diseases & 3.00 & 1.31 & 0.01 & 0.70 & 0.63 & 0.69 \\
\hline Liver diseases & 5.75 & 3.11 & 0.01 & 1.39 & 1.88 & 0.81 \\
\hline Cancer & 0.44 & 0.15 & 0.02 & 0.48 & 0.36 & 0.33 \\
\hline Number of medicines taken & 1.65 & 0.22 & $<0.01$ & 1.43 & 0.54 & 0.34 \\
\hline Confine to bed & 5.80 & 5.80 & $<0.01$ & 13.88 & 20.17 & 0.07 \\
\hline Stop activities during heatwaves & 0.71 & 0.19 & $<0.01$ & 1.28 & 0.96 & 0.75 \\
\hline Use an air-conditioner & 0.11 & 0.04 & $<0.01$ & 0.27 & 0.23 & 0.12 \\
\hline Use refreshment & 0.05 & 0.02 & $<0.01$ & 0.10 & 0.11 & 0.03 \\
\hline ADL impaired $(=1)$ & 0.03 & 0.01 & $<0.01$ & $2.14 \mathrm{e}+08$ & $4.38 e+11$ & 0.10 \\
\hline Having more social activities & 0.07 & 0.02 & $<0.01$ & 0.11 & 0.09 & 0.01 \\
\hline
\end{tabular}

Bold typeface indicates significance at $p \leq 0.05$ in the multiple regression.

${ }^{*}$ ORs with $p<0.05$ estimated by simple logistic regression included in the AOR regression models.

ADL, Activities of Daily Living; AOR, adjusted OR.

Table 3 Adjusted ORs (AORs) estimated by multiple conditional logistic regression*

\begin{tabular}{lccl}
\hline & OR & SE & (95\% Cl) \\
\hline $\begin{array}{l}\text { More social activities } \\
\text { Use refreshment }\end{array}$ & 0.11 & 0.09 & $(0.02$ to 0.57$)$ \\
$\begin{array}{l}\text { Having emergency } \\
\text { buttons }\end{array}$ & 0.10 & 0.11 & $(0.01$ to 0.84$)$ \\
$\begin{array}{l}\text { Higher education level } \\
\text { Air-conditioner in }\end{array}$ & 0.11 & $(0.01$ to 0.96$)$ \\
bedroom & 0.12 & 0.18 & $(0.23$ to 0.99$)$ \\
$\begin{array}{l}\text { Heart disease } \\
\text { Dementia }\end{array}$ & 13.56 & 16.39 & $(1.27$ to 144.86$)$ \\
\hline
\end{tabular}

${ }^{*}$ Factors selected with the best goodness of fit (pseudo $\mathrm{R}^{2}=0.85$ ).

would respond to heat. ${ }^{24}$ Yet there is little evidence to support a protective effect from behaviour change during a heatwave. Evidence suggests that personal perceptions of health risks from heatwaves could be a crucial determinant in shaping behaviour change strategies during heatwaves. ${ }^{16}$ However, our findings do not prevent the sending of messages to individuals regarding the potential health benefit from some behaviour changes during heatwaves.

Our results suggest that a higher socioeconomic status (eg, a higher education level and income level) would be a protective factor for reducing morbidity during heatwaves. Poverty is an important determinant of risk of heat stroke mortality in the USA during heatwaves, but little or no effect of deprivation on morbidity risk was reported by most studies from the 2003 heatwave in Europe. ${ }^{25}$ People with a lower socioeconomic status and lower education level may have fewer chances to access and understand useful information and warnings about heat and health. In addition, poorer people are more likely to have a poorer health status and live in areas and accommodation that do not provide respite during heat. They may not have air-conditioning, or may be reluctant to run it due to the cost; also, they may be less likely to go to cooler venues such as shopping centres. ${ }^{15}$

Most population-based heatwave studies are located in cities, but there are indications that the heat island effect in cities increases the risk of health effects during 
extreme heat events. ${ }^{26}$ Individual living conditions have so far not been sufficiently examined. The benefit of having and/or using air-conditioners during heatwaves is not consistently supported in the literature. ${ }^{12}$ In South Australia and France, having an air-conditioner at home was reported as a protective factor for adverse health outcomes during heat. ${ }^{8} 18$ Our study indicates that having air-conditioner in bedrooms may reduce the risk of having direct heat-related morbidity by $>80 \%$ during heatwaves in Adelaide. Air-conditioner is not commonly used in the study region despite extreme summers being a common experience, particularly in the case group ( $17 \%$ and $52 \%$ in our cases and controls, respectively). There may be additional risk factors from living conditions based on studies in America and Europe, for example, lack of insulation, north-facing building, living alone and living on the top floor of a building (although this factor is not an issue in Adelaide because of the low population density). ${ }^{18} 2728$ However, these studies are mainly focused on deaths rather than hospitalisations during heat, and the spectrum of risk factors for individual living conditions is therefore likely to differ.

The single-variable analysis suggests that several coexisting illnesses, including kidney disease, heart disease, dementia and depression, could be related to an increased risk of having heat-related hospitalisation. After controlling for other variables, the final multiple logistic regression model indicates that the risk of direct heat-related hospitalisations could be 10 times higher during heatwaves for those having a heart disease, and $>20$ times for those having dementia, compared with those who do not have these conditions. The vulnerability to heat for those who have a heart disease has been addressed by several studies, both in Australia and other countries. ${ }^{4529-31}$ Our findings are consistent with these studies in terms of the increased risks during heat among those who had pre-existing heart conditions. The estimated figures for increased risks varied a lot in previous studies, which may be due to different data sources, age differences in the study population, definitions of exposure to heat and the choices of statistical models. The salient increase in the risk of having direct heat-related hospital admissions for patients with heart disease in our study is probably more reliable due to the collection of individual data in a case-control design, unlike the previous studies that used ecological study designs with population data.

There is insufficient evidence of adverse health outcomes during heatwaves among patients with dementia in recent studies. ${ }^{32}$ Our findings suggest a significantly increased risk of hospitalisations for those who live with dementia during heatwaves, which means potentially demanding healthcare would be needed for these patients in such extreme heat events. Patients with dementia may alter their risk perceptions and be less able to undertake changes in behaviour that could help them cope with the heat; may be less able to call for help when distressed; and may be on medications which interfere with thermoregulation. ${ }^{32} 33$ Our study may be the first to report a dramatically increased risk of morbidity among patients with dementia during heatwaves, with potentially significant implications for the development of guidelines for the care of patients with dementia during extreme heat events.

Kidney disease and depression are not statistically significant in our final multiple regression model, which may be due to the small sample size or the higher rate of comorbidities among the participants (eg, 12\% of all participants had both kidney disease and heart disease and $13 \%$ had both depression and heart disease). However, given the significant ORs for kidney disease and depression in the simple regression analysis and findings from previous studies regarding the negative association between the conditions and adverse health outcomes during heatwaves, ${ }^{6}{ }^{7}$ it might be useful to intervene in these patients during heatwaves even if we are not sure if it is because of the particular diseases that they are at risk or the generally poor health status due to comorbidities.

A major strength of our study is the matched casecontrol study design, with data of potential risk factors collected at an individual level, thus providing a stronger evidence base than previous ecological studies using data at a population level. The limitations of a casecontrol study are mainly due to recall bias. However, we have reviewed patients' hospital medical records to increase the accuracy of individual information, including their diagnoses and some sociodemographic data, as recorded by nurses when being admitted to hospitals. We could not examine the interaction of comorbidities because of the complexity in biological causal associations among these coexisting conditions. There might also be limitations from recruiting $60 \%$ of eligible patients and we could not have a full comparison between those who refused to participate with the participants due to incomplete records.

In conclusion, by analysing individual data, we are able to detect that pre-existing heart disease and dementia may substantially increase the risk of direct heat-related hospitalisations, and that the presence of an air-conditioner in the bedroom, more social activities, a higher education level and use of refreshment and emergency buttons could reduce the risk of hospitalisation during heatwaves. Since direct heat-related illness is largely avoidable, our findings will benefit the development of health policies, clinical practices, community resilience and further interventional research in order to reduce morbidity in a warming climate.

Author affiliations

${ }^{1}$ School of Public Health, China Studies Centre, University of Sydney, Sydney, New South Wales, Australia

${ }^{2}$ South Australian Department for Health and Ageing (DHA), Adelaide, South Australia, Australia

${ }^{3}$ School of Public Health, University of Adelaide, Adelaide, South Australia Australia

${ }^{4}$ Global Health Institute, Duke University and Duke Kunshan University, Shanghai, China 
${ }^{5}$ School of Biological Sciences, University of Adelaide, Adelaide, South Australia, Australia

${ }^{6}$ Department of Clinical Pharmacology, Royal Adelaide Hospital, Adelaide, South Australia, Australia

Contributors All the authors contributed to the study design and funding application. YZ, AK and MN contributed to data collection. YZ, GT and KD contributed to data analyses. YZ, MN and PB drafted the initial manuscript. All the authors contributed to the interpretation of results, as well as the writing and editing of the manuscripts.

Funding This work was supported by the Australian Research Council Linkage Project and the Department for Health and Ageing South Australia (LP110200820). YZ was an Australian National Health and Medical Research Council Public Health Training Fellow (602749).

\section{Competing interests None declared.}

Ethics approval This project was approved by the Human Research Ethics Committees (HREC) at the University of Adelaide (H-101-2010) and the South Australian Department for Health and Ageing (DHA) (376/06/2013).

Provenance and peer review Not commissioned; externally peer reviewed.

Data sharing statement The original data can only be accessed by the research investigators working at the South Australia Department of Health, which was required by the ethical approvals. The corresponding authors can be contacted if the de-identified dataset is required.

Open Access This is an Open Access article distributed in accordance with the Creative Commons Attribution Non Commercial (CC BY-NC 4.0) license, which permits others to distribute, remix, adapt, build upon this work noncommercially, and license their derivative works on different terms, provided the original work is properly cited and the use is non-commercial. See: http:// creativecommons.org/licenses/by-nc/4.0/

\section{REFERENCES}

1. IPCC. Climate Change 2013. The 5th Assessment Report. New York, 2013.

2. Australian Bureau of Meteorology. State of climate report 2014. Canberra, 2014.

3. Li M, Gu S, Bi P, et al. Heat waves and morbidity: current knowledge and further direction-a comprehensive literature review. Int $J$ Environ Res Public Health 2015;12:5256-83.

4. Gronlund CJ, Zanobetti A, Schwartz JD, et al. Heat, heat waves, and hospital admissions among the elderly in the United States, 19922006. Environ Health Perspect 2014;122:1187-92.

5. Nitschke M, Tucker GR, Hansen AL, et al. Impact of two recent extreme heat episodes on morbidity and mortality in Adelaide, South Australia: a case-series analysis. Environ Health 2011;10:42. http:// www.ehjournal.net/content/10/1/42.

6. Hansen A, Bi P, Nitschke M, et al. The effect of heat waves on mental health in a temperate Australian city. Environ Health Perspect 2008;116:1369-75

7. Hansen AL, Bi P, Ryan P, et al. The effect of heat waves on hospital admissions for renal disease in a temperate city of Australia. Int $J$ Epidemiol 2008;37:1359-65.

8. Hansen $A, B i$ P, Pisaniello $D$, et al. Heat-health behaviours of older people in two Australian states. Australas J Ageing 2015;34:E19-25.

9. Hanson-Easey S, Bi P, Walker I, et al. Public understandings of climate change and adaptation in South Australia. National Climate Change Adaptation Research Facility (NCCARF) Report. Australia, 2013.

10. Williams S, Bi P, Newbury J, et al. Extreme heat and health: perspectives from health service providers in rural and remote communities in South Australia. Int $J$ Environ Res Public Health 2013;10:5565-83.

11. Xiang J, Bi P, Pisaniello D, et al. The impact of heatwaves on workers' health and safety in Adelaide, South Australia. Environ Res 2014;133:90-5

12. Hajat S, O'Connor M, Kosatsky T. Health effects of hot weather: from awareness of risk factors to effective health protection. Lancet 2010;375:856-63.

13. Ye X, Wolff R, Yu W, et al. Ambient temperature and morbidity: a review of epidemiological evidence. Environ Health Perspect 2012;120:19-28.

14. Reid CE, O'Neill MS, Gronlund CJ, et al. Mapping community determinants of heat vulnerability. Environ Health Perspect 2009;117:1730-6

15. Kovats RS, Hajat S. Heat stress and public health: a critical review. Annu Rev Public Health 2008;29:41-55.

16. Wolf $\mathrm{J}$, Adgera WN, Lorenzonia I, et al. Social capital, individual responses to heat waves and climate change adaptation: an empirical study of two UK cities. Glob Environ Change 2010;20:44-52.

17. Semenza JC, Rubin $\mathrm{CH}$, Falter $\mathrm{KH}$, et al. Heat-related deaths during the July 1995 heat wave in Chicago. N Engl J Med 1996;335: 84-90.

18. Vandentorren S, Bretin P, Zeghnoun A, et al. August 2003 heat wave in France: risk factors for death of elderly people living at home. Eur J Public Health 2006;16:583-91.

19. Kilbourne EM, Choi K, Jones T, et al. Risk factors for heatstroke: a case-control study. JAMA 1982;247:3332-6.

20. Canouï-Poitrine F, Cadot E, Spira A Groupe Régional Canicule. Excess deaths during the August 2003 heat wave in Paris, France. Rev Epidemiol Sante Publique 2006;54:127.

21. Zhang Y, Nitschke M, Bi P. Risk factors for direct heat-related hospitalization during the 2009 Adelaide heatwave: a case crossover study. Sci Total Environ 2013;442:1-5.

22. Nitschke M, Tucker $\mathrm{G}$. The unfolding story of heat waves in metropolitan Adelaide. Adelaide: SA Health Report, 2010.

23. Dupont W. Power calculations for matched case-control studies. Biometrics 1988;44:1157-68.

24. Kalkstein AJ, Sheridan SC. The social impacts of the heat-health watch/warning system in Phoenix, Arizona: assessing the perceived risk and response of the public. Int $J$ Biometeorol 2007:52:43-55.

25. Gabriel KMA, Endlicher WR. Urban and rural mortality rates during heat waves in Berlin and Brandenburg, Germany. Environ Pollut 2011:159:2044-50.

26. Abrahamson V, Wolf $\mathrm{J}$, Lorenzoni I, et al. Perceptions of heatwave risks to health: interview-based study of older people in London and Norwich, UK. J Public Health (Oxf) 2009;31:119-26.

27. Naughton MP, Henderson A, Mirabelli MC, et al. Heat-related mortality during a 1999 heat wave in Chicago. Am J Prev Med 2002;22:221-7.

28. Poumadère M, Mays C, Le Mer S, et al. The 2003 heat wave in France: dangerous climate change here and now. Risk Anal 2005;25:1483-94.

29. Tian Z, Li S, Zhang J, et al. Ambient temperature and coronary heart disease mortality in Beijing, China: a time series study. Environ Health 2012;11:56.

30. Schwartz J, Samet J, Patz J. Hospital admissions for heart disease: the effects of temperature and humidity. Epidemiology 2004;15:755-61.

31. Liang WM, Liu WP, Chou SY, et al. Ambient temperature and emergency room admissions for acute coronary syndrome in Taiwan. Int J Biometeorol 2008;52:223-9.

32. Åström DO, Bertil F, Joacim R. Heat wave impact on morbidity and mortality in the elderly population: a review of recent studies. Maturitas 2011;69:99-105.

33. Garssen J, Harmsen C, De Beer J. The effect of the summer 2003 heat wave on mortality in the Netherlands. Euro Surveill 2005;10:165-8. 